\title{
Quantum Zeno suppression of three-body losses in Bose-Einstein condensates
}

\author{
R. Schützhold and G. Gnanapragasam \\ Fakultät für Physik, Universität Duisburg-Essen, D-47048 Duisburg, Germany
}

\begin{abstract}
We study the possibility of suppressing three-body losses in atomic Bose-Einstein condensates via the quantum Zeno effect, which means the delay of quantum evolution by frequent measurements. It turns out that this requires very fast measurements with the rate being determined by the spatial structure of the three-body form factor, i.e., the point interaction approximation $\delta^{3}\left(\mathbf{r}-\mathbf{r}^{\prime}\right)$ is not adequate. Since the molecular binding energy $E_{b}$ provides a natural limit for the measurement rate, this suppression mechanism can only work if the form factor possesses certain special properties.

PACS numbers: $03.65 . \mathrm{Xp}, 34.50 . \mathrm{Bw}, 34.10 .+\mathrm{x}$
\end{abstract}

To the best of our knowledge, Bose-Einstein condensates of trapped dilute alkali gases are the coldest systems in the Universe [1]. Apart from their low temperature, atomic Bose-Einstein condensates are an ideal laboratory for studying many-body quantum effects [2, 3] because they can be well manipulated and controlled experimentally by using lasers and magnetic fields [4]. However, since the gaseous state is not the real ground state of these atoms, inelastic collisions due to three-body recombination occur [5]. In such an exothermic process, three atoms collide to form a dimer and an unbound atom, which carries away the excess energy and momentum. The binding energy thus released is converted into the kinetic energies of the colliding partners (molecule and third atom) which leave the trap. As a result of this process, the condensate is susceptible to loss and heating [6, 7], thereby inevitably limiting its lifetime and the minimum achievable temperature. Consequently, one is lead to the question as to how one could suppress this undesired loss mechanism.

In this work we address this important question using the idea of quantum Zeno effect which is basically the delay or even inhibition of the time evolution of a quantum state by performing frequent measurements [810]. As an example, consider a simple two-level system undergoing Rabi oscillations of frequency $\omega$. When the initial state $\left|\psi_{1}\right\rangle$ gets rotated under the action of the total Hamiltonian $\hat{H}$, the state evolves to

$$
|\psi(t)\rangle=e^{-i \hat{H} t}\left|\psi_{1}\right\rangle=\cos \omega t\left|\psi_{1}\right\rangle-i \sin \omega t\left|\psi_{2}\right\rangle .
$$

Without measurements, the probability to find the state in $\left|\psi_{1}\right\rangle$ after time $t$ is $P(t)=\left|\left\langle\psi_{1}\left|e^{-i H t}\right| \psi_{1}\right\rangle\right|^{2}=\cos ^{2} \omega t$. When $N$ measurements are made during this time (after equidistant time intervals), the survival probability reads

$$
P_{N}(t)=[P(t / N)]^{N} \approx \exp \left(-\frac{t^{2}}{N \tau^{2}}\right)
$$

where $1 / \tau^{2}=\left\langle\psi_{1}\left|\hat{H}^{2}\right| \psi_{1}\right\rangle=\omega^{2}$ [1]. For large $N$, the survival probability $P_{N}(t)$ approaches one, i.e., under continuous measurements the time evolution of the system is prevented. Thus, quoting [11] Zeno's quantum- mechanical arrow (the wave function), sped by the Hamiltonian, does not move, if it is continuously observed.

The above example demonstrates the suppression of transitions between two discrete levels in a quantum system. However, the aforementioned three-body losses correspond to a decay into a continuum of states. Thus, we have to study whether and when the quantum Zeno effect occurs in such cases. To this end, we consider 12 . the following model Hamiltonian $(\hbar=1)$

$$
\hat{H}=E_{0}|g\rangle\langle g|+\int d k\left[E_{k}|k\rangle\langle k|+\left(g_{k}|g\rangle\langle k|+\text { h.c. }\right)\right],
$$

where $\langle g \mid g\rangle=1,\left\langle k \mid k^{\prime}\right\rangle=\delta\left(k-k^{\prime}\right)$, and $\left\langle g \mid k^{\prime}\right\rangle=0$. Here $|g\rangle$ denotes an initial quasi-bound state (i.e., a resonance) with energy $E_{0}$ coupled to a continuum of states $|k\rangle$ with energies $E_{k}$ via the coupling strength $g_{k}$. Similar Hamiltonians have been used to study, for example, a two-level atom interacting with radiation field [13].

To the lowest order in perturbation $g_{k}$, the total decay probability $P_{d}(t)=\int d k\left|\left\langle k\left|e^{-i \hat{H} t}\right| g\right\rangle\right|^{2}$, takes the form

$$
P_{d}(t)=t^{2} \int d E|g(E)|^{2} \frac{\sin ^{2}\left[\left(E-E_{0}\right) t / 2\right]^{2}}{\left[\left(E-E_{0}\right) t / 2\right]^{2}},
$$

where we have used $|g(E)|^{2}=\left|g_{k}\right|^{2}\left(d E_{k} / d k\right)^{-1}$. For short times, we obtain a quadratic growth of the decay probability $P_{d}(t) \propto t^{2}$ similar to the previous example in Eq. (11). Consequently, the time evolution can be delayed via the quantum Zeno effect according to Eq. (2). For longer times (but still within the region of perturbation theory), the decay probability grows linearly $P_{d}(t) \propto t$ and we recover Fermi's golden rule. In this regime, measurements would not slow down the time evolution significantly. Therefore, the crossover time $T_{*}$ when the transition between these two different regimes takes place [20], i.e., $P_{d}\left(t \ll T_{*}\right) \propto t^{2}$ and $P_{d}\left(t \gg T_{*}\right) \propto t$, determines how fast one has to measure in order to slow down the decay via the quantum Zeno effect. We may estimate the crossover time $T_{*}$ via calculating the intersection point of the two asymptotic expressions $P_{d}\left(t \ll T_{*}\right) \propto t^{2}$ and $P_{d}\left(t \gg T_{*}\right) \propto t$ which yields

$$
\frac{1}{T_{*}}=\mathcal{O}\left(\int d E \frac{|g(E)|^{2}}{\left|g\left(E_{0}\right)\right|^{2}}\right)=\mathcal{O}(\Delta E) .
$$


This is reminiscent of Heisenberg's energy-time uncertainty relation and implies that one has to measure before the energy conservation begins to play a role in order to observe the quantum Zeno effect.

Alternatively, using the wave-packet description one may write $T_{*}$ in the following way

$$
\frac{1}{T_{*}}=\mathcal{O}\left(v_{\text {group }} \Delta k\right)=\mathcal{O}\left(\frac{v_{\text {group }}}{\Delta r}\right) .
$$

Here $\Delta k$ and $\Delta E$ are the widths of $\left|g_{k}\right|^{2}$ and $|g(E)|^{2}$, respectively, and $v_{\text {group }}=d E_{k} / d k$ is the group velocity (such that $v_{\text {group }} \Delta k \approx \Delta E$ ). For early times, the wave- packet created by the decay of the initial quasi-bound state $|g\rangle$ into the continuum of states $|k\rangle$ possesses the width $\Delta k$ in momentum space and thus it spreads over $\Delta r=\mathcal{O}(1 / \Delta k)$ in position space. Thus, $T_{*}$ is the period during which this wave-packet of spatial size $\Delta r$ needs to move away with its group velocity $v_{\text {group }}$. In other words, as long as the wave-packet is close to its initial position (and thus has large overlap with the original quasi-bound state $|g\rangle$ ), its decay can be suppressed via the quantum Zeno effect - once the wave-packet has crossed its own dimensions and moved away, measuring does not change much anymore.

Studying the simple toy model in Eq. (3), we found that the quantum Zeno effect may also occur in the decay to a continuum of states, but only if measurements are performed fast enough - with the characteristic time $T_{*}$ being determined by the form factor $\left|g_{k}\right|^{2}$. Now let us study a more realistic model Hamiltonian for three-body losses in Bose-Einstein condensates. For simplicity, we restrict our consideration to one species of atoms with mass $m$ (described by the field operator $\hat{\psi}$ ) coupled to one species of molecules with mass $2 m$ (and the field operator $\hat{\phi}$ )

$$
\hat{H}=\int d^{3} r\left\{\hat{\psi}^{\dagger}(\mathbf{r})\left(\frac{-\nabla^{2}}{2 m}\right) \hat{\psi}(\mathbf{r})+\hat{\phi}^{\dagger}(\mathbf{r})\left(\frac{-\nabla^{2}}{4 m}+E_{b}\right) \hat{\phi}(\mathbf{r})+\int d^{3} r^{\prime}\left[W\left(\mathbf{r}-\mathbf{r}^{\prime}\right) \hat{\phi}^{\dagger}\left(\frac{\mathbf{r}+\mathbf{r}^{\prime}}{2}\right) \hat{\psi}\left(\mathbf{r}^{\prime}\right) \hat{\psi}(\mathbf{r})+\text { h.c. }\right]\right\} .
$$

Here $E_{b}$ is the negative molecular binding energy and $W\left(\mathbf{r}-\mathbf{r}^{\prime}\right)$ is the atom-molecule coupling. Since the characteristic length scales of molecule formation are typically much smaller than the trap size, we assume a homogeneous condensate with density $\rho$ and omit the trap potential $V(\mathbf{r})$. Within standard perturbation theory (i.e., expansion into powers of the coupling strength $W$ ) we recover the usual Feynman diagrams depicted in Fig. 1. Three-body losses are described by processes of third (or higher) order (i.e., Fig. 1 1 ) and the decay probability reads to lowest order in $W$

$$
P_{d}(t)=\rho^{3}|\tilde{W}(0)|^{2} \int d^{3} k|\tilde{W}(\mathbf{k})|^{2}|\tilde{W}(2 \mathbf{k})|^{2}\left|\frac{3}{4 E_{b}} \frac{e^{i \omega_{1} t}-1}{\omega_{1} \omega_{3}}+\frac{1}{\omega_{3}-\omega_{2}}\left(\frac{3}{4} \frac{e^{i \omega_{2} t}-1}{\omega_{2} \omega_{3}}-\frac{e^{i \omega_{3} t}-1}{E_{b} \omega_{3}}\right)\right|^{2} .
$$

Here $\tilde{W}(\mathbf{k})=\tilde{W}(k)$ is the Fourier transform of $W\left(\mathbf{r}-\mathbf{r}^{\prime}\right)$ and we have abbreviated $\omega_{1}=E_{b}+3 E_{k} / 2, \omega_{2}=E_{b}-E_{k} / 2$, $\omega_{3}=3 E_{k} / 2$, and $E_{k}=k^{2} /(2 m)$. Initially, at time $t=0$, we consider a situation $|\Psi(t=0)\rangle=\left|\Psi_{0}\right\rangle$ where all the atoms have Bose condensed $\hat{\psi}\left|\Psi_{0}\right\rangle=\sqrt{\rho}\left|\Psi_{0}\right\rangle$ and there are no molecules $\hat{\phi}\left|\Psi_{0}\right\rangle=0$. Of course, this is a strong simplification (assuming perfect measurements and weak depletion etc.) but the main results should remain unaffected.

The expression for the time dependence of the decay probability in Eq. (8) allows us to study the occurrence of the quantum Zeno effect. For small $t$, a Taylor expansion of the integrand in Eq. (8) in powers of $t$ yields

$$
P_{d}(t)=\left(\frac{\rho^{3}|\tilde{W}(0)|^{2}}{36} \int d^{3} k|\tilde{W}(\mathbf{k})|^{2}|\tilde{W}(2 \mathbf{k})|^{2}\right) t^{6} .
$$

Thus, the decay probability increases as $t^{6}$ initially. This departure from the conventional $t^{2}$ behavior obtained earlier is attributed to the three-body loss as a thirdorder process. In this regime, frequent measurements slow down the decay significantly.

For large $t$, on the other hand, we recover the linear time dependence analogous to Fermi's golden rule: In this limit, the points of stationary phase in the integrand dominate the integral in Eq. (8). Since the binding energy $E_{b}$ is negative, we have $\omega_{2}<0, \omega_{3}>0$ and thus $\omega_{3}>\omega_{2}$ for all $k>0$. Consequently, the only point of stationary phase (which is also the only point where one of the denominators vanishes) is located at $\omega_{1}=0$ and (as one would expect) corresponds to energy conservation $3 E_{k} / 2=-E_{b}$. Thus we derive the asymptotic (large $t$ ) behavior of Eq. (8) in complete analogy to Eq. (4): For large values of $t$, only the term containing $\sin ^{2}\left(\omega_{1} t / 2\right) / \omega_{1}^{2}$ exhibits a peak at $\omega_{1}=0$, of height $\propto t^{2}$ and width $\propto 1 / t$. Thus the significant contribution to the integral arises from the neighborhood of this peak. Performing the integration we get (for large $t$ )

$$
P_{d}(t)=\frac{256 \pi^{2} \rho^{3} m^{5}}{27 k_{0}^{7}}\left|\tilde{W}(0) \tilde{W}\left(k_{0}\right) \tilde{W}\left(2 k_{0}\right)\right|^{2} t,
$$

where the value of $k_{0}=\left(4 m E_{b} / 3\right)^{1 / 2}$ is dictated by energy conservation.

The crossover time $T_{*}$ between the two regimes (9) and (10) can again be estimated by equating these two 
asymptotic expressions for $P_{d}(t)$

$$
\frac{1}{T_{*}^{5}}=\mathcal{O}\left(\frac{3 k_{0}^{7}}{1024 \pi^{2} m^{5}} \int d^{3} k\left|\frac{\tilde{W}(\mathbf{k}) \tilde{W}(2 \mathbf{k})}{\tilde{W}\left(k_{0}\right) \tilde{W}\left(2 k_{0}\right)}\right|^{2}\right) .
$$

The integral in the equation above scales with the cubed width $k_{W}^{3}$ of the function $\tilde{W}(\mathbf{k})$ in $k$-space (relative to its value at $k_{0}$ and $2 k_{0}$, respectively). Thus, the crossover time $T_{*}$ can be estimated as

$$
\frac{1}{T_{*}^{5}}=\mathcal{O}\left(\frac{k_{W}^{3} k_{0}^{7}}{m^{5}}\right)=\mathcal{O}\left(E_{b}^{5} \frac{k_{W}^{3}}{k_{0}^{3}}\right) .
$$

Similar to the previous example in Eq. (3), this expression facilitates an intuitive interpretation - even though it is more qualitative than the quantitative picture described after Eq. (6). At early times, the spatial size $\Delta r$ of the molecular wave-packet scales with $\Delta r=\mathcal{O}\left(1 / k_{W}\right)$ while its group velocity is determined by $k_{0}$, i.e., the binding energy $E_{b}$. Hence $T_{*}$ grows for both $k_{W}$ and/or $k_{0}$ shrinking.
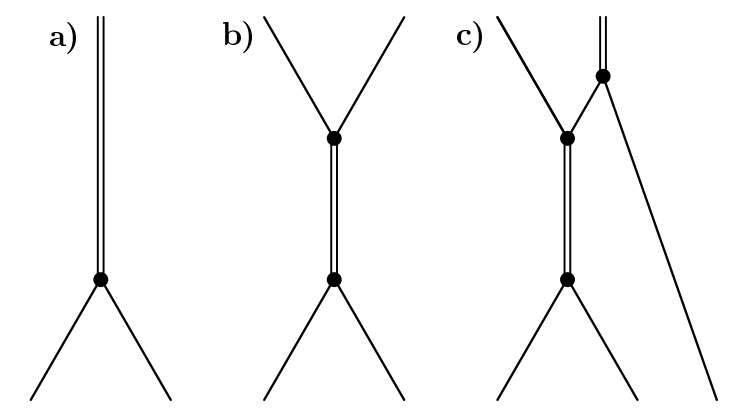

FIG. 1: Feynman diagrams for the Hamiltonian in Eq. (7). Single lines denote atoms and double lines are molecules while the black dot is the interaction vertex. The left diagram a) shows the first-order process of direct molecule formation which is forbidden by energy conservation. The middle diagram b) corresponds to atom-atom scattering via an intermediate ("virtual") molecular state, which is a second-order effect. Finally, the right diagram c) combines the processes a) and b) and shows the actual third-order three-body recombination process resulting in the formation of a real molecule plus an atom (carrying away the excess energy and momentum).

In units of the inverse binding energy $E_{b}$, the crossover time $T_{*}$ scales with the ratio $k_{0} / k_{W}$ to the power $3 / 5$. In order to achieve a significant delay of the time evolution via the quantum Zeno effect, we would have to measure, i.e., detect the formed molecule, faster than this time scale $T_{*}$. The presence of the molecule could be detected via driving a light induced transition between molecular bound states which is off-resonant for all atomic states (see also 14]). Then the energy-time uncertainty relation implies that this measurement cannot be done faster than the inverse binding energy $E_{b}$. Even if we could imagine a detection method for which this energy-time uncertainty bound does not apply, it would be unwise to measure faster than the inverse binding energy $E_{b}$ : For such measurements, the direct two-body recombination process in Fig. 1h which is forbidden by energy conservation, would start to contribute. Since the rate of this first-order process (if allowed) is typically much larger than that of the third-order effect in Fig. 1k, the condensate would become even more unstable. Therefore, stabilizing the Bose-Einstein condensate via the quantum Zeno effect is only possible if $T_{*} E_{b} \gg 1$. With Eq. (12) we see that this requires $k_{0} \gg k_{W}$. So if the structure of $\tilde{W}(\mathbf{k})$ is basically determined by just one length scale (e.g., the $s$ wave scattering length $a_{s}$ ) as in Fig. $2 \mathrm{~b}$, we cannot stabilize the Bose-Einstein condensate via the quantum Zeno effect. However, if $\tilde{W}(\mathbf{k})$ has a more complicated, e.g., spiky, structure as in Fig. 2b, we could have $k_{0} \gg k_{W}$ and thus it could be possible.
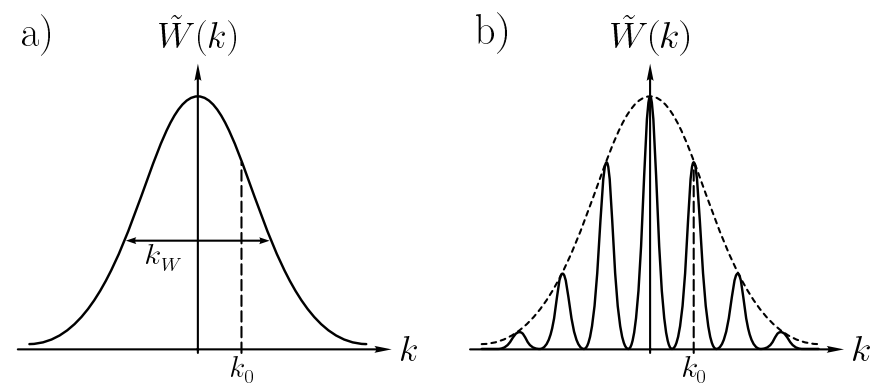

FIG. 2: Sketch of two possible forms for the atom-molecule coupling $\tilde{W}$ as a function of $k$ illustrating the two characteristic cases: In the left picture a), $\tilde{W}$ has no sub-structure and thus $k_{0}$ and $k_{W}$ are of the same order of magnitude $k_{W} \sim k_{0}$, i.e., the quantum Zeno effect is not applicable. (Note that $k_{W} \gg k_{0}$ would still be possible in this case - but in this limit, the quantum Zeno effect does also not apply.) In the right picture b), the function $\tilde{W}$ has more structure and thus we have $k_{W} \ll k_{0}$, which enables us to apply the quantum Zeno effect.

In summary, we studied the possibility of suppressing three-body recombinations in dilute atomic BoseEinstein condensates via the quantum Zeno effect. This fascinating phenomenon corresponds to the delay or even inhibition of quantum evolution by repeated measurements if they are fast enough. Addressing the question of how fast is fast enough, we first see that the probability for a positive measurement outcome (saying that the system has actually evolved) must always be small. In addition to this trivial bound, another timescale arises in the decay of a meta-stable state into a continuum: In such a set-up, we obtain a quadratic (or higher polynomial) growth of the decay probability at early times ("quantum Zeno regime") and a linearly increasing decay probability at later times ("Fermi golden rule regime"). Obviously, measurements can only slow down the evolution during the initial period (i.e., the "quantum Zeno regime"). For the model Hamiltonian in 
Eq. (3), describing the tunnelling of a particle out of a potential well, for example, the crossover time $T_{*}$ separating the two regimes [20] is given by the width of the corresponding wave-packet in comparison with its group velocity. In the case of three-body recombinations in dilute atomic Bose-Einstein condensates, this crossover time $T_{*}$ is determined by the spatial structure of the three-body form factor $W\left(\mathbf{r}-\mathbf{r}^{\prime}\right)$ in Eq. (7). As a result, the often used [15, 16] point interaction approximation $W\left(\mathbf{r}-\mathbf{r}^{\prime}\right) \propto \delta^{3}\left(\mathbf{r}-\mathbf{r}^{\prime}\right)$ is not adequate for this question. Since the molecular binding energy $E_{b}$ provides a natural limit for the measurement rate, this suppression mechanism can only work if the typical width $k_{W}$ of the form factor $\tilde{W}$ is much smaller than characteristic momentum of the final molecule/atom $k_{0}=\left(4 m E_{b} / 3\right)^{1 / 2}$ [17].

It might be illustrative to compare our approach with the work [14] in which the inhibition of three-body recombination via resonant $2 \pi$ laser pulses was studied. First of all, it should be noted that these $2 \pi$ laser pulses considered in [14] do not correspond to an actual measurement but induce a phase shift. Therefore, the mechanism studied in [14] is not the quantum Zeno effect, but corresponds to passive error correction techniques known in quantum information theory under the names "spinecho" or "bang-bang" method, see, e.g., [19]. Nevertheless, both phenomena are based on the crucial difference between summing amplitudes and probabilities in quantum theory. Therefore, the repetition rate of the $2 \pi$ laser pulses considered in [14] should lie in the "quantum Zeno regime" in order to induce a significant slow-down (in the "Fermi golden rule regime" they would have very little effect), i.e., similar temporal constraints should apply in both cases.

RS acknowledges support from DFG (SFB-TR12). GG is indebted to Prof. S. Thomae for general fruitful discussions, kind help with the figures and hospitality.

[1] M. H. Anderson, J. R. Ensher, M. R. Matthews, C. E. Wieman, and E. A. Cornell, Science 269, 198 (1995).
[2] W. Ketterle, Phys. Today 52, 30 (1999).

[3] J. R. Anglin and W. Ketterle, Nature 416, 211 (2002).

[4] E. A. Cornell and C. E. Wieman, Rev. Mod. Phys. 74, 875 (2002), and references therein.

[5] E. Nielsen and J. H. Macek, Phys. Rev. Lett. 83, 1566 (1999).

[6] J. Dziarmaga and K. Sacha, Phys. Rev. A 68, 043607 (2003).

[7] T. Weber, J. Herbig, M. Mark, H.-C. Nägerl, and R. Grimm, Phys. Rev. Lett. 91, 123201 (2003).

[8] B. Misra and E. C. G. Sudarshan, J. Math. Phys. 18, 756 (1977).

[9] W. M. Itano, D. J. Heinzen, J. J. Bollinger, and D. J. Wineland, Phys. Rev. A 41, 2295 (1990).

[10] M. C. Fischer, B. Gutiérrez-Medina, and M. Raizen, Phys. Rev. Lett. 87, 040402 (2001).

[11] P. Facchi and S. Pascazio, J. Phys. A: Math. Theor. 41, 493001 (2008).

[12] P. Facchi, H. Nakazato, and S. Pascazio, Phys. Rev. Lett. 86, 2699 (2001).

[13] M. Frasca, Ann. Phys. 306, 193 (2003), and references therein.

[14] C. P. Search, W. Zhang, and P. Meystre, Phys. Rev. Lett. 92, 140401 (2004).

[15] E. Timmermans, P. Tommasini, R. Côté, M. Hussein, and A. Kerman, Phys. Rev. Lett. 83, 2691 (1999).

[16] S. J. J. M. F. Kokkelmans and M. J. Holland, Phys. Rev. Lett. 89, 180401 (2002).

[17] It has been shown that the recombination into loosely bound molecules, with the pseudopotential interaction and a positive $s$-wave scattering length $a_{s}$, obeys universal features in the sense that all relevant quantities are unique functions of $a_{s}$. For example, the binding energy $E_{b}$ of the $s$-wave bound state takes the simple form $E_{b}=-1 /\left(m a_{s}^{2}\right)$ [18] from which it follows that $k_{0}=2 / \sqrt{3} a_{s}$. If this universality also extends to the internal structure of $W$, then the quantum Zeno effect is not applicable in this case.

[18] R. A. Duine and H. T. C. Stoof, Phys. Rep. 396, 115 (2004); T. Köhler and K. Góral, Rev. Mod. Phys. 78, 1311 (2006).

[19] M. S. Byrd and D. A. Lidar, Quant. Info. Proc. 1, 19 (2002).

[20] Note that the crossover time $T_{*}$ in Eqs. (5) and (11) should not be confused with the time scale $\tau$ in Eq. (2) which is sometimes [11] called "quantum Zeno time". 